Supplement of Earth Surf. Dynam., 7, 911-927, 2019

https://doi.org/10.5194/esurf-7-911-2019-supplement

(C) Author(s) 2019. This work is distributed under

the Creative Commons Attribution 4.0 License.

(c) (1)

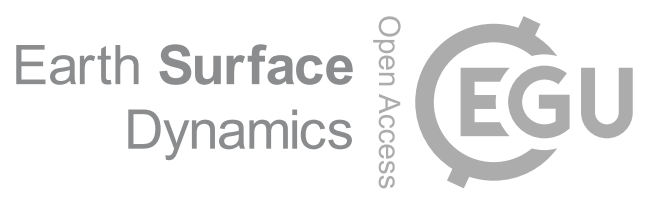

Supplement of

\title{
How does the downstream boundary affect avulsion dynamics in a laboratory bifurcation?
}

\section{Gerard Salter et al.}

Correspondence to: Gerard Salter (salte040@umn.edu)

The copyright of individual parts of the supplement might differ from the CC BY 4.0 License. 


\section{Contents}

1. Figures $\mathrm{S} 1$ to $\mathrm{S} 4$

2. Table S1

3. Text $\mathrm{S} 1$

\section{Introduction}

Our supplementary information is organized as follows: First, we provide four supporting figures. Figure S1 shows time series of the delta front positions for each experiment. Figure S2 shows the time evolution of the bed elevation profiles for experiments 4 and 5. Figure S3 shows an example of bar migration from experiment 5. Figure S4 provides the final water/bed topography around the bifurcation point for experiment 5. Finally, Figure S5 and Table S1 show our estimates of the Shields stress, and 10 should be viewed in the context of Text S1, which describes the methodology and limitations of calculating the Shields stress. 

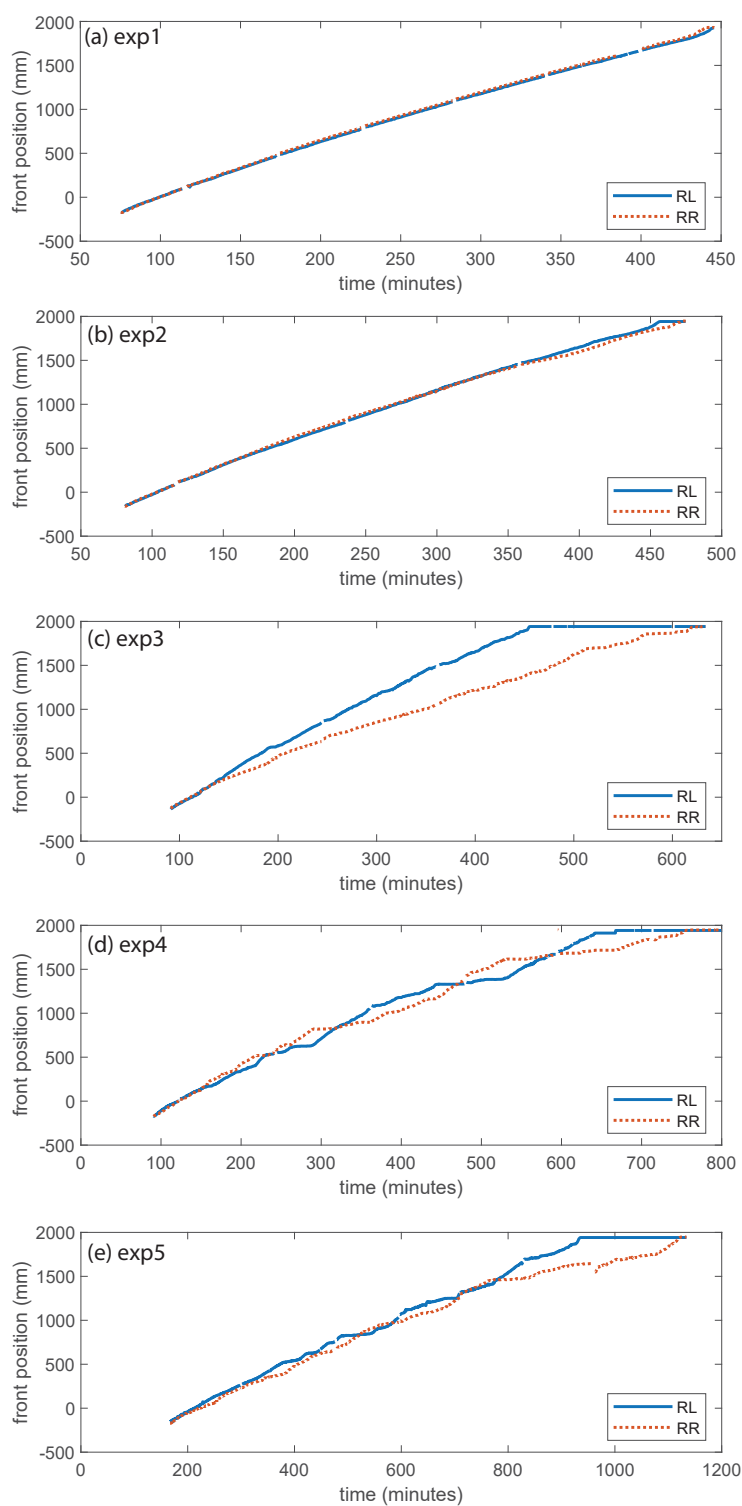

Figure S1. Position of delta fronts (topset/foreset slope break) over time for each of the five experiments. A position of 0 is defined as the location where the branches split. The method for extracting the delta front positions is described in the main paper. 

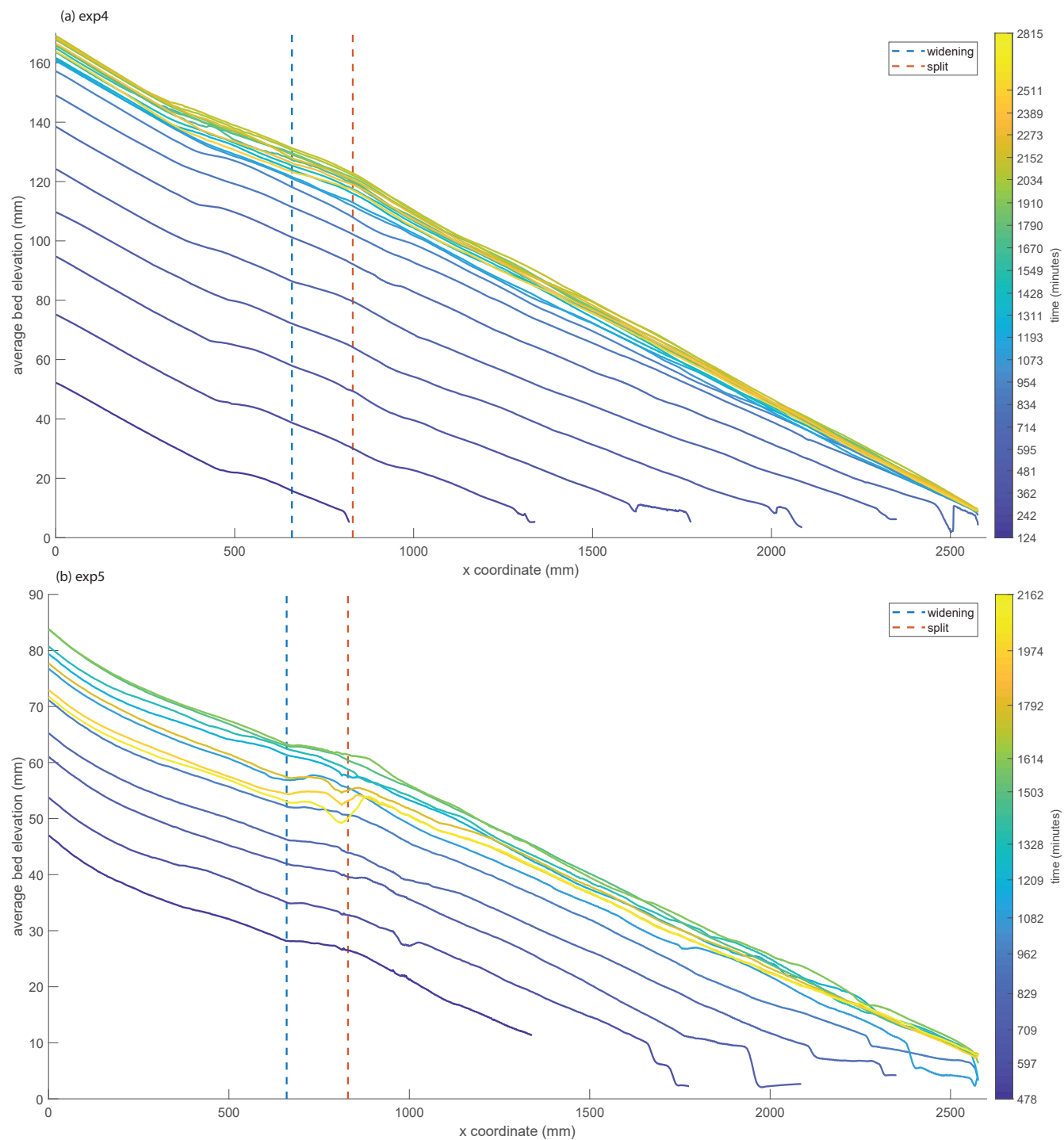

Figure S2. Time evolution of bed elevation profiles for (a) experiment 4 and (b) experiment 5. Profile colors indicate the time at which topographic scan was taken. Each point in the profile is an average of all bed elevations at that given $x$ position, where $x$ is the cartesian coordinate oriented parallel to the upstream channel direction, and increases with distance downstream. For reference, the dotted vertical lines indicate the start of the widening portion of the bifurcation flume, and the position of the nose/split. Note the $1 \mathrm{~cm}$ of variability in bed elevation in experiment 4 after start of bypass phase upstream of the split, linked to bypass dynamics discussed in the main paper. For experiment 5 , note that the final profile is significantly lower than the maximum elevation profile; erosion coincided with consolidation of the flow into a single channel. 


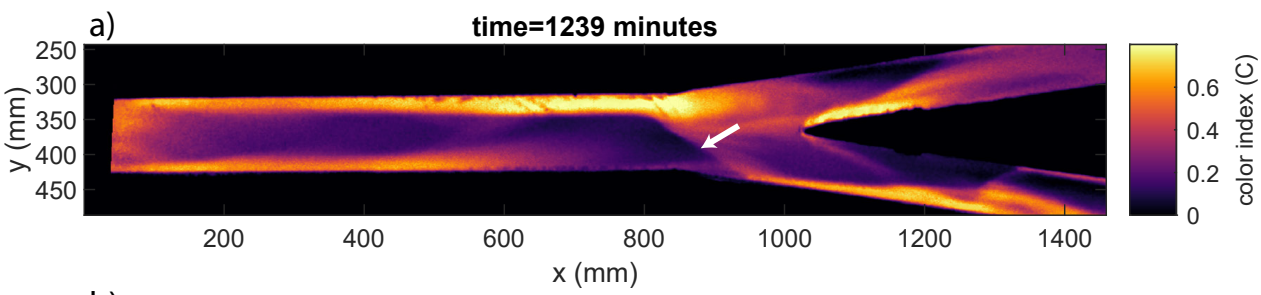

b)

time=1240 minutes

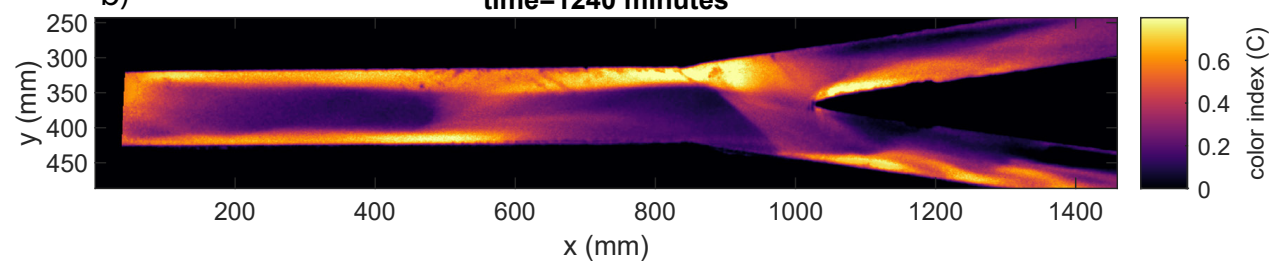

c) time=1241 minutes
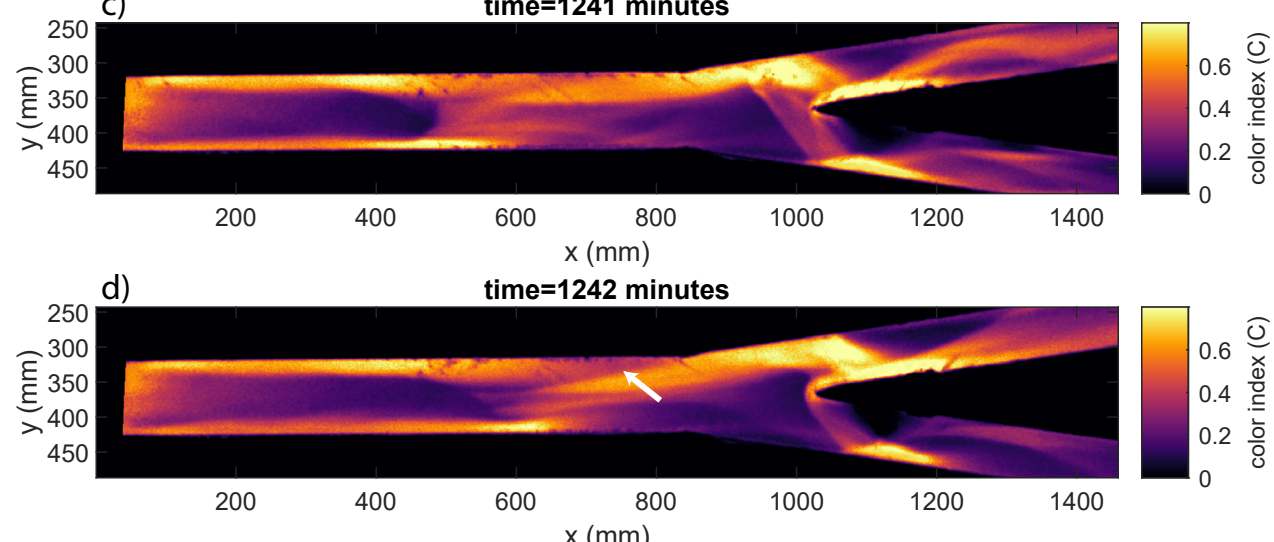

e)

unsersanames

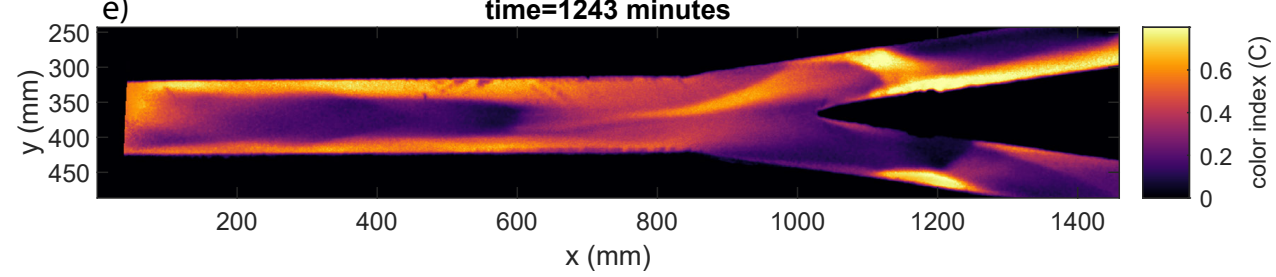

Figure S3. Overhead images from experiment 5 at 1-minute intervals, showing bar migration on the timescale of minutes. White arrows in subfigures a) and d) point to bar fronts. 


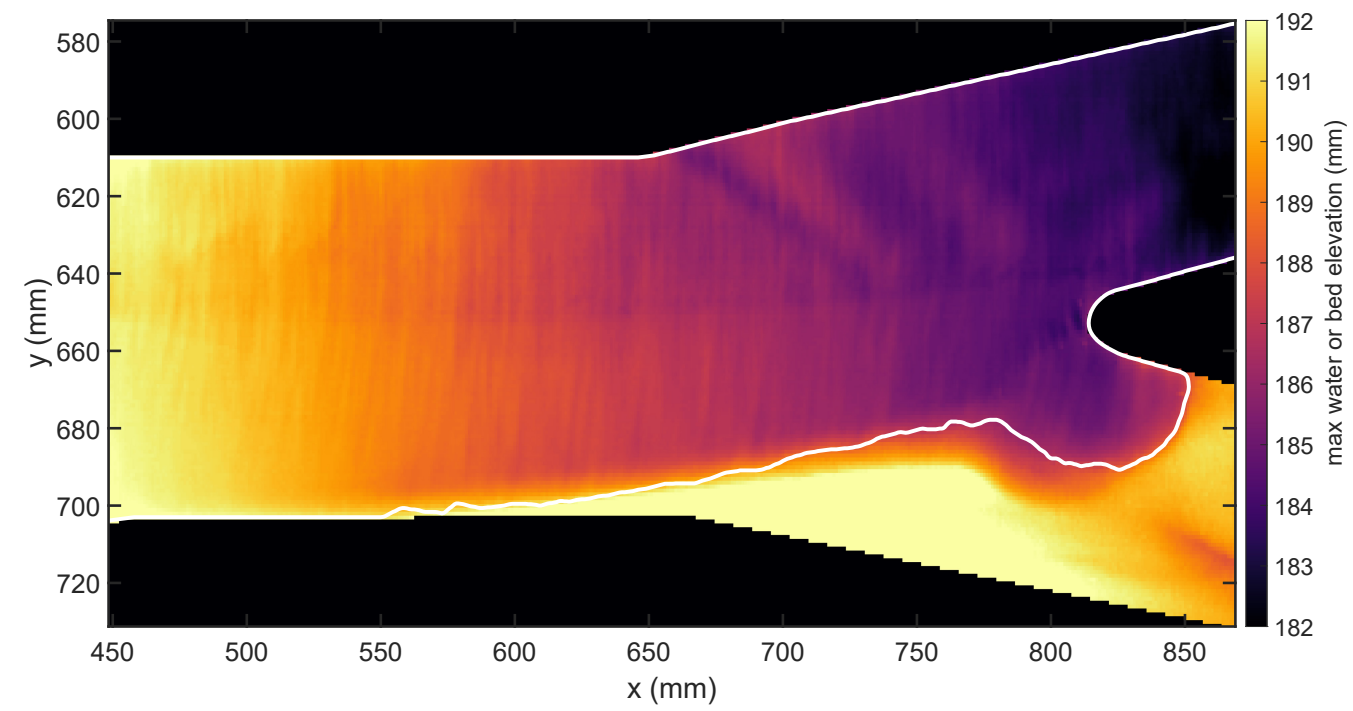

Figure S4. Detail of the bifurcation using final bed and water elevations from experiment 5. Flow direction is from left to right, Image shows water elevation in wetted regions, and bed elevation in dry regions. The solid white line delineates the land/water interface; the river right branch is completely dry. The bed elevation of the river right branch is several millimeters higher than the water surface elevation immediately upstream. 

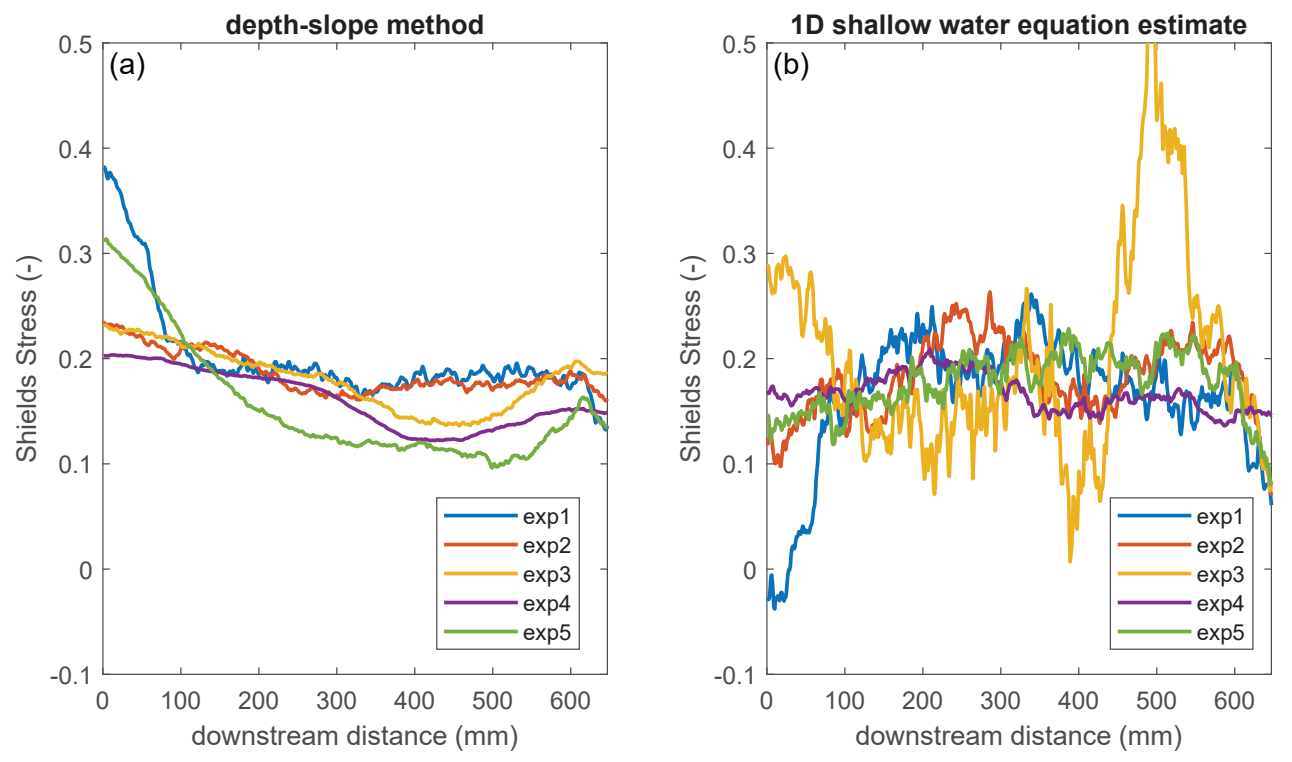

Figure S5. Local estimates of the Shields stress in the upstream channel. Profiles have been smoothed with a moving average window of 10 $\mathrm{cm}$. Downstream edge of figure is the start of the bifurcation widening. (a) Shields stress profiles estimated using equation (S.5). (b) Shields stress profiles estimated using equation (S.6). As explained below in Text S1, the sediment flux values reported in the main paper should be regarded as more reliable than the Shields stress estimates. 


\begin{tabular}{|c|c|c|}
\hline Experiment & Shields stress (eq. S.5) & Shields stress (eq. S.6) \\
\hline $\exp 1$ & 0.25 & 0.12 \\
$\exp 2$ & 0.22 & 0.14 \\
$\exp 3$ & 0.21 & 0.19 \\
$\exp 4$ & 0.19 & 0.17 \\
$\exp 5$ & 0.23 & 0.15 \\
\hline
\end{tabular}

Table S1. Estimates of the Shields stress averaged over the upstream $20 \mathrm{~cm}$. 


\section{Text S1 Estimation of the Shields Stress}

In our paper, we chose to report the dimensionless sediment flux as opposed to the Shields stress, because sediment flux is the independent variable we directly controlled. However, previous studies on bifurcations used the upstream channel Shields stress instead. As discussed below, our dimensionless sediment flux measurement is more reliable than our Shields stress estimates.

5 Here, we report estimates of the Shields stress for each of our experiments, and discuss some of the complexities in obtaining these estimates.

The Shields stress is defined as

$$
\tau_{*}=\frac{\tau_{0}}{\left(\rho_{s}-\rho\right) g d_{s}}
$$

where $\tau_{0}$ is the bed shear stress, given by

$10 \tau_{0}=\rho c_{f} u^{2}$

where $c_{f}$ is the dimensionless bed resistance coefficient, and $u$ is the depth-averaged velocity. Assuming steady flow, the depth-averaged velocity is:

$u=\frac{Q_{w}}{W h}$

We can write the 1D shallow water equation as:

$15 \rho u^{2} \frac{\partial h}{\partial x}+\rho g h S_{w}=\rho c_{f} u^{2}$

where $x$ is the streamwise coordinate, and $S_{w}$ is the water surface slope. We see that the right-hand side of the equation is the shear stress (from equation S.2), and therefore, so is the left-hand side of the equation.

It is common to assume normal flow, in which case the first term of equation (S.4) is assumed to be small, and we recover the familiar depth-slope product:

$\tau_{0}=\rho g h S_{w}$

Note that under normal flow conditions, the bed and water surfaces are parallel.

Alternatively, if we do not neglect the downstream gradient in depth, we obtain:

$\tau_{0}=\rho g h S_{w}+\rho u^{2} \frac{\partial h}{\partial x}$

We note that in reality, the depth is not uniform in a channel cross-section, so in place of the depth $h$, we use the hydraulic radius $h_{R}$. Similar results are obtained using the average depth, given that our channels are relatively wide.

To compute the Shields stress, we obtain a local estimate for each cross-section in the upstream $20 \mathrm{~cm}$ of our scan, and average those values. Our estimates, using both equations (S.5) and (S.6), are shown in Table S1. We use only the upstream portion of the domain, because further downstream the depth is less uniform in the cross-flow direction. Due to the non-linearity of sediment transport, if the Shields stress in a cross-section is variable, then a sediment transport estimate using the average Shields stress will underestimate the actual sediment transport. An additional reason is that 2-dimensional effects (i.e. crossstream flow, induced for example by bars) are not accounted for in equation (S.6). We find that our estimates of the Shields stress are lower using equation (S.6) rather than (S.5), indicating that spatial accelerations cannot be neglected. We also note that $\exp 5$ has the lowest sediment feed rate of our experiments, but based on either estimate does not have a lower Shields stress, as would be predicted.

35 We obtain Shields stress estimates using both equations (S.5) and (S.6). In Figure S5, we plot the Shields stress longitudinal profiles for the bypass portions of our experiments. Under bypass conditions, the sediment flux should be uniform on average in 
the longitudinal direction, and therefore, so should the Shields stress. We observe in Figure S5 (a), the Shields stress decreases from upstream to downstream over the first $20 \mathrm{~cm}$, most notably in experiments 1 and 5 (the two highest water discharge experiments). Figure S5 (b) shows that using the full equation (S.6) corrects for the over-prediction of the Shields stress in the upstream reach for experiment 5, but over-corrects experiment 3, at least within the first $10 \mathrm{~cm}$. We also note that because 5 equation (S.6) involves the derivative of the water depth, the noise in the profiles is greatly enhanced. Experiment 3 in particular has large fluctuations in the Shields stress that we do not believe are real, based on our argument that the sediment flux should be uniform from upstream to downstream.

Given the complexity of estimating the Shields stress as described above, and given that the dimensionless sediment flux is directly imposed, we believe that the dimensionless sediment flux is the more reliable measurement to report in our main 10 paper, as opposed to the Shields stress. Again, from a theoretical vantage point, the Shields stress and dimensionless sediment flux are directly linked; setting the value of one implies a value for the other. 